\title{
Salivary stress biomarkers and anxiety symptoms in children with and without temporomandibular disorders
}

\section{Fernanda Yukie KOBAYASHI(a) Maria Beatriz Duarte GAVIÃO(a) Maria Carolina Salomé MARQUEZIN(a) \\ Fernando Luiz Affonso FONSECA ${ }^{(\text {b) }}$ Ana Bheatriz Marangoni MONTES(c) Taís de Souza BARBOSA(d) Paula Midori CASTELO(b)}

(a) Universidade de Campinas - Unicamp, Piracicaba Dental School, , Department of Pediatric Dentistry University of Campinas, Piracicaba, SP, Brazil.

(b) Universidade Federal de São Paulo Unifesp, Department of Pharmaceutical Sciences, Diadema, SP, Brazil.

(c) Universidade do Oeste Paulista - Unoeste, Department of Pediatric Dentistry, Presidente Prudente, SP, Brazil.

(d) Universidade Federal de Juiz de Fora - UFJF, Department of Odontology, Governador Valadares, MG, Brazil.

Declaration of Interest: The authors certify that they have no commercial or associative interest that represents a conflict of interest in connection with the manuscript.

\section{Corresponding Author:}

Paula Midori Castelo

E-mail:pcastelo@yahoo.com

https://doi.org/10.1590/1807-3107BOR-2017.vol31.0078

Submitted: Feb 21, 2017

Accepted for publication: July 27, 2017

Last revision: Aug 10, 2017
Abstract: The etiology of temporomandibular disorders (TMD), which are considered as a heterogeneous group of psychophysiological disturbances, remains a controversial issue in clinical dentistry. This study aimed to evaluate whether the salivary alpha-amylase (sAA), cortisol levels, and anxiety symptoms differ between children with and without TMD. Initially, 316 young subjects were screened in public schools (nonreferred sample); 76 subjects aged 7-14 years were selected and comprised the TMD and control groups with 38 subjects each matched by sex, age, and the presence/absence of sleep bruxism. Four saliva samples were collected: upon waking, $30 \mathrm{~min}$ and $1 \mathrm{~h}$ after awakening (fasting), and at night (at $8 \mathrm{PM}$ ) on 2 alternate days to examine the diurnal profiles of cortisol and sAA. Anxiety symptoms were screened using the Multidimensional Anxiety Scale for Children (MASC-Brazilian version). Shapiro-Wilk test, Student's t-test/Mann-Whitney U test, and correlation tests were used for data analysis. No significant differences were observed in the salivary cortisol area under the curve $\left(\mathrm{AUC}_{\mathrm{G}}\right.$ mean $\pm \mathrm{SD}=90.22 \pm 63.36 \times 94.21 \pm 63.13 \mu \mathrm{g} / \mathrm{dL} / \mathrm{min})$ and $\mathrm{sAA} \mathrm{AUC}_{\mathrm{G}}$ (mean $\pm \mathrm{SD}=2544.52 \pm 2142.00 \times 2054.03 \pm 1046.89 \mathrm{U} / \mathrm{mL} / \mathrm{min}$ ) between the TMD and control groups, respectively $(p>0.05)$; however, the clinical groups differed in social anxiety domain $(\mathrm{t}=3.759 ; \mathrm{CI}=2.609$, 8.496), separation/panic $(t=2.243 ; C I=0.309,5.217)$, physical symptoms $(\mathrm{U}=433.500)$, and MASC total score $(\mathrm{t}=-3.527 ; \mathrm{CI}=-23.062,-6.412)$, with a power of the test $>80 \%$ and large effect size $(d=0.80)$, with no significant correlation between the MASC total score, cortisol, and sAA levels. Although children with TMD scored higher in anxiety symptoms, no difference was observed in the salivary stress biomarkers between children with and without TMD.

Keywords: Temporomandibular Joint Disorders; Biomarkers; Saliva; Alpha-Amylases

\section{Introduction}

Temporomandibular disorders (TMD) are characterized by clinical signs and symptoms involving the temporomandibular joints (TMJs), masticatory muscles, bones, and associated tissues. ${ }^{1}$ In children, one in six children and adolescents has signs of TMJ disorders, ${ }^{2}$ and limited 
mouth opening, clicking, crepitation, TMJ and muscle pain are the most frequent signs and symptoms observed. ${ }^{1,3}$ Recent studies reported that symptoms increase during adolescence, are prominent in the middle age, and then gradually diminish, ${ }^{4}$ with females being more affected. ${ }^{5,6}$

TMD is considered as a heterogeneous group of psychophysiological disorders. Some conditions and habits, such as bruxism, nail biting, and nonnutritive sucking are common in children, and these may contribute to the TMD manifestation. ${ }^{6}$ Depression and anxiety are also considered as risk factors, ${ }^{7}$ and individuals affected by these disorders may experience negative impacts on their social and emotional welfare, general well-being, and academic performance, ${ }^{8}$ which may activate their physiological stress systems. ${ }^{1}$

Anxiety is defined as a persistent anticipation or apprehension regarding one or more situations to which a person is exposed; ${ }^{9}$ herein, it is essential to differentiate between the state anxiety, which is a transitory and emotional condition, and trait anxiety, which is a stable personality characteristics of the potential for manifesting state anxiety. ${ }^{10}$ Stress, in contrast, is a response to the threatening, uncontrollable, or unexpected situations, ${ }^{9}$ which can be measured by salivary biomarkers, such as cortisol and alpha-amylase (sAA) ${ }^{11}$

Little data exists on the possible relationship between stress and TMD. Past studies revealed the possible predisposing, triggering and/or worsening role of some psychological factors, ${ }^{12}$ and a high comorbidity with illness and other pain conditions has been observed. ${ }^{5}$ Children and adolescents with lower socioeconomic status presented higher rates of recurrent pain (headache, abdominal/back pain), and an association between abdominal pain and headache, headache and dizziness, and back pain and dizziness was found. ${ }^{13}$ Some behaviors, especially those that increase the muscle tension, and emotional states may exacerbate the symptoms of TMD. ${ }^{14}$

Repeated exposure to stressful situations can trigger overactivation of the hypothalamus-pituitary-adrenal (HPA) axis, increasing the cortisol levels, ${ }^{15}$ which may have detrimental effects on health. Previous studies found increased cortisol levels in females with TMD when compared with the controls. ${ }^{14,16}$
sAA is a digestive and antimicrobial enzyme, which increases under stressful conditions and is known to induce catecholamine production, thus reflecting the sympathetic activity. ${ }^{11}$ The literature reveals that psychiatric disorders and psychosocial stress increase sAA secretion; ${ }^{17,18}$ in addition, adults with TMD may reveal impairments in the sympathetic-adrenergic component of the autonomic system, which may influence pain symptoms and catecholamine responses at rest and during stress. ${ }^{19,20}$

Each risk factor for TMD needs to be considered to appropriately diagnose and plan the management strategies. ${ }^{1}$ Stress is considered as an important factor in the onset and maintenance of musculoskeletal disorders, although little is known about the relationship between TMD, stress, and anxiety in children. The hypothesis to be tested was whether children with TMD reveal increased diurnal levels of salivary stress biomarkers and anxiety symptoms. Thus, this study aimed to evaluate the sAA and cortisol levels and the anxiety symptoms in pediatric subjects with TMD, when compared with the matched controls.

\section{Methods}

\section{Study design and ethical considerations}

This research was approved by the Research Ethics Committee of the Piracicaba Dental School, University of Campinas (UNICAMP), Brazil (Protocol No. 004/10), and its reporting follows the STROBE recommendations for observational/case-control studies. Each subject and his/her parent/guardian gave voluntary consent to participate in this research by signing an assent form and a parental/guardian consent form, respectively.

\section{Sample}

Sample size was calculated based on the results found in the literature, ${ }^{18,21,22}$ considering $80 \%$ power and an alpha level of 0.05 for comparing between independent samples (case $\times$ control group). According to the results, we presumed that about 19-31 subjects would be required in each group to evaluate the salivary cortisol levels, ${ }^{21,22}$ and 17 subjects were needed in each group to evaluate the sAA levels. ${ }^{18}$ Since a wide interindividual variability is observed 
in evaluating the salivary biomarkers, we opted to include a larger sample.

This study tentatively screened 316 caries-free children and adolescents between 6 and 17 years in 4 public schools of Piracicaba, Brazil (August-November 2011). Of these, 191 subjects met the inclusion criteria, and a convenient nonreferred sample of 76 subjects was selected (age range 7-14 years), with 38 subjects (24 females and 14 males) in each group (TMD and control groups).

The inclusion and exclusion criteria were assessed during the interview and clinical examination. The inclusion criterion involved children in the mixed or permanent dentition phases. The exclusion criteria were: presence of dental caries; premature tooth loss; tooth shape/number/structure anomalies; oral soft tissue abnormalities or trauma; use of dental prostheses; pain of dental origin; previous/current orthodontic treatment; any orofacial pain condition that could interfere with the diagnosis of TMD; general systemic disturbances (diabetes, hypertension, and others); current use of medications (e.g., antidepressants, muscle relaxants, narcotic or nonsteroidal anti-inflammatory drugs); neurological or psychiatric disorders previously diagnosed; and children who did not cooperate with the research procedures.

\section{Clinical and physical examinations}

The clinical examinations were conducted at schools in a reserved room using mirror, artificial LED light, and a probe. The clinical signs of TMD were assessed using the research diagnostic criteria for temporomandibular disorders (RDC/TMD) by a calibrated examiner (FYK), based on a series of clinical protocols and procedures and strict diagnostic criteria for the most common types of TMD. ${ }^{23}$ Two diagnostic axes are contemplated in this protocol: Axis I establishes a diagnosis based on the clinical variables, while Axis II establishes a diagnosis based on the psychological variables. As Axis II was not validated for children and due to the age of the participants (unable to provide reliable answers for the mentioned questionnaire), only Axis I was applied.

The control group comprised children with no sign or symptom of TMD, and they were matched with the TMD group for age, sex, and the presence of sleep bruxism, as these are the potential factors for differences in salivary cortisol and sAA levels. ${ }^{24,25}$

The signs and symptoms of sleep bruxism were recorded considering the minimal diagnostic criteria, ${ }^{26}$ the sibling/parental report of tooth grinding sounds (at least thrice a week), and the presence of shiny and polish facets on the incisors and/or first permanent molars (based primarily on palatal surface and incisal edges and working cusps, respectively). ${ }^{25}$

Body weight and height were determined using an anthropometric scale, and the body mass index $\left(\mathrm{BMI}=\mathrm{Kg} / \mathrm{m}^{2}\right)$ was calculated .

\section{Evaluation of anxiety symptoms}

Anxiety symptoms were evaluated using the Brazilian version of Multidimensional Anxiety Scale for Children (MASC), using 39 questions (Likert), which are intended to examine the subject's feelings, thoughts, and actions. The MASC has four subfactors: physical symptoms, harm avoidance, social anxiety, and separation/panic. For each item, the children were asked to check the number that indicated the frequency with which the affirmative was true for him/her: 0 (never true about me) to 3 (often true about $\mathrm{me}$ ). The questionnaire was answered by the subject himself/herself and was individually applied by the researcher, to clear any doubt regarding the items, and to avoid reading difficulties for the results. ${ }^{27} \mathrm{This}$ study did not intend to diagnose anxiety, and raw data of the total scale and each subfactor were used as a continuous variable.

\section{Saliva collection}

Four home-stimulated saliva samples were collected on two alternate weekdays to examine the diurnal secretion profile of cortisol and sAA. Subjects (and their parents) were instructed to wake at $7 \mathrm{AM}$, and children were asked to gently chew the swab (salivettes, Sarstedt, Numbrecht, Germany) for 2 min, sufficient to enable the roll to be soaked in saliva. The first, second, third, and fourth samples were collected while lying in bed (on waking), 30 min after awakening ( $+30 \mathrm{~min}$, fasting), $1 \mathrm{~h}$ after awakening ( $+1 \mathrm{~h}$, fasting), and at night $(8 \mathrm{PM})$ on each day, respectively. The samples were stored in a refrigerator and delivered to the researcher at school 
the following day. Salivettes were transported to the laboratory on ice on the same day and centrifuged (at $3500 \mathrm{rpm}$ for $5 \mathrm{~min}$ ); furthermore, saliva samples were divided into four aliquots (two for sAA and two for cortisol) and were stored at $-80{ }^{\circ} \mathrm{C}$ until analysis. According to Granger et al., ${ }^{28}$ salivary samples can be stored for at least $24 \mathrm{~h}$ at room temperature or at $4{ }^{\circ} \mathrm{C}$ (refrigerator) without compromising the integrity of sAA measurement. The recommendations of the cortisol immunoassay kit (Salimetrics ${ }^{\circledR}$, State College, PA, USA) were also followed for salivary sample handling, as described below.

Subjects were instructed not to perform physical exercises or ingest caffeinated beverages on the day prior to saliva collection, and abstain from food, beverages, and toothbrushing prior sampling. ${ }^{25}$ Participant nonadherence to the study protocol was avoided by providing complete information about the purpose and reinforcing the procedures (by telephone call), 1 day before collection. Moreover, each parent/guardian was asked to provide details regarding the collection timings.

\section{Salivary cortisol analyses}

Salivary cortisol levels were assayed in duplicate using a commercial, highly sensitive enzyme immunoassay kit (Salimetrics ${ }^{\circledR}$, State College, PA, USA) by one researcher (FYK). After thawing, the samples were centrifuged at $1500 \times \mathrm{g}$ for $15 \mathrm{~min}$ and whole saliva $(25 \mathrm{ml})$ was added to each well of a microtiter plate and read at $450 \mathrm{~nm}$ in a microplate reader (Stat Fax 2100, Awareness Tech. Inc., Palm City, FL, USA). Enzyme-linked immunosorbent assays (ELISAs) were performed according to the manufacturer's instructions. The minimum concentration of cortisol that can be distinguished from 0 was $0.003 \mu \mathrm{g} / \mathrm{dL}^{25}$

\section{Salivary alpha-amylase quantification}

sAA levels were analyzed on a different day after cortisol analysis by an automated technique (Flexor E6002-190 Automated Clinical Chemistry Analyzer, Vital Scientific, Dieren, Switzerland), at the Clinical Analyses Laboratory of ABC Medical School (Santo André, Brazil). sAA concentrations were measured using the enzymatic method in diluted saliva (1:25) (ELI Tech, Seppim S.A., SEES, France). Samples of known concentrations provided by the Brazilian Society of Clinical Pathology and Laboratory Medicine were used as the standard to calibrate the automated system, and the parameters adopted were: sAA level $=61.3 /$ range of $50.3-72.3 \mathrm{U} / \mathrm{mL}^{.29}$

\section{Measurement errors}

To assess the method error of the clinical variables (TMD signs and wear facets of sleep bruxism), Kappa tests were used on data collected by one examiner (FYK) from 20 subjects aged 6-11 years who were not included in the study on two separate occasions, with an interval of 14 days.

\section{Statistical analysis}

Statistical analysis was performed using BioEstat 5.3 (Mamirauá, Belém, PA, Brazil) and SigmaPlot 13 (Systat Software Inc., San Jose, CA, USA) statistical packages with a $5 \%$ significance level by one of the authors (PMC, Applied Statistics Specialist). Shapiro-Wilk test reported that the distributions of the BMI, cortisol, and sAA data deviated from normality. There were no missing data. Differences in BMI were tested between groups using Mann-Whitney U test.

sAA activity and cortisol concentrations were evaluated by calculating the area under the curve against time $\left(\mathrm{AUC}_{\mathrm{G}}\right)$, which was estimated by the trapezoid method respective to the ground level ${ }^{30}$ for each day, and the final value comprised the mean of both. The cortisol awakening response (CAR) was calculated by subtracting the cortisol concentration upon awakening from cortisol concentration measured 30 min after awakening.

Due to their skewed distributions, sAA and cortisol data were transformed to more accurate normality (square root transformation and natural $\log$ "ln," respectively)..$^{28}$ Two sample t-test was used to test the differences in cortisol $\mathrm{AUC}_{\mathrm{G}}$, $\mathrm{sAA} \mathrm{AUC} \mathrm{C}_{\mathrm{G}}$ and CAR between the TMD and control groups. The correlation between salivary cortisol and $\mathrm{SAA} \mathrm{AUC}_{\mathrm{G}}$ was evaluated by means of Spearman correlation test.

The MASC total and subfactor scores (continuous variables) were compared between groups using two sample t-test or Mann-Whitney U test, where appropriate. Cohen's d effect size was calculated using the formula: ${ }^{31}$ 
$d=\left(M_{1}-M 2\right) / \sqrt{ }\left(S D_{1}^{2}+S D_{2}^{2}\right) / 2$

A correlation matrix examined the correlation between the MASC total score, cortisol, and sAA salivary levels.

\section{Results}

During evaluation of the method error, kappa coefficient obtained for the mouth opening was considered almost perfect (0.92), and kappa value for pain on palpation on the right masseter was equal to 0.67 , indicating a substantial agreement. Signs of sleep bruxism (wear facets) presented a good level of reliability, with a kappa coefficient of 0.77 (substantial agreement).

A total of 316 caries-free children and adolescents were screened, and finally 76 participants were selected. The subjects in the TMD group $(n=38)$ were diagnosed based on: myofascial pain (IA, $n=28$ ), disk displacement with reduction (IIA, $\mathrm{n}=4$ ); myofascial pain with limited opening $(\mathrm{IB}, \mathrm{n}=5)$, and disk displacement without reduction (IIC, $\mathrm{n}=1$ ).

The sample characteristics in accordance with the paired clinical groups are presented in Table 1. BMI did not differ between groups $(p=0.787$;
Mann-Whitney U test). The diurnal salivary cortisol and sAA profiles for both groups are depicted in Figures 1 and 2, respectively.

Statistical analyses revealed that clinical groups did not differ in the salivary cortisol and $\mathrm{SAA} \mathrm{AUC} \mathrm{G}_{\mathrm{G}}$ (t-test; $\mathrm{CI}=-0.436-0.260$ and $\mathrm{CI}=-3.589-11.276$, respectively; transformed data). Moreover, $\mathrm{CAR}$ did not differ between groups ( $\mathrm{t}$-test; $\mathrm{CI}=-0.090-0.034$; raw data). The Spearman correlation coefficients obtained for the salivary cortisol and SAA AUC were neither significant for the TMD group $($ rho $=-0.03, \mathrm{p}>0.05)$ nor for the control group (rho $=0.17, \mathrm{p}>0.05$ ).

Clinical groups significantly differed in the MASC total score (t-test; $\mathrm{t}=-3.527 ; \mathrm{CI}=-23.062--6.412$ ) and the following subfactors' scores: social anxiety (t-test; $\mathrm{t}=$ 3.759; CI = 2.609-8.496), separation/panic ( $\mathrm{t}$-test; $\mathrm{t}=2.243 ; \mathrm{CI}=0.309-5.217)$, and physical symptoms (Mann-Whitney U test; $\mathrm{U}=433.500$; Table 2), with a power of the test above $80 \%$ and large Cohen's effect size $(\mathrm{d}=0.80)$. No significant correlation was observed between the MASC total score and the salivary cortisol and sAA levels (control group: $r h o=0.085$ and rho $=-0.136$, respectively; TMD group: rho $=0.040$ and rho $=0.048$, respectively).

Table 1. Characteristics of the sample according to clinical groups.

\begin{tabular}{|c|c|c|c|}
\hline Variable & TMD group & Control group & $p$-value \\
\hline $\mathrm{N}$ & 38 & 38 & NA \\
\hline \multicolumn{4}{|l|}{ Age (y) } \\
\hline Mean (SD) & $10.63(1.68)$ & $10.63(1.68)$ & NA \\
\hline Median (IQR) & $11.00(1.00)$ & $11.00(1.00)$ & NA \\
\hline Age range & 7-14 & 7-14 & NA \\
\hline $\operatorname{Sex}(n)$ & $24 q 14 \delta^{\lambda}$ & $24 q 14 \hat{\jmath}$ & NA \\
\hline \multicolumn{4}{|l|}{ BMI $\left(\mathrm{Kg} / \mathrm{m}^{2}\right)$} \\
\hline Mean (SD) & $19.41(4.90)$ & $19.36(4.41)$ & - \\
\hline Median (IQR) & $18.28(6.35)$ & $18.35(5.71)$ & $0.787^{*}$ \\
\hline Presence of sleep bruxism & 7 (yes) 31 (no) & 7 (yes) 31 (no) & NA \\
\hline \multicolumn{4}{|c|}{ Salivary amylase $A \cup C_{G}(U / m L / m i n)$} \\
\hline Mean (SD) & $2544.52(2142.00)$ & 2054.53 (1046.89) & $0.306^{* *}$ \\
\hline Median (IQR) & $1873.94(1575.83)$ & $2044.03(1183.60)$ & - \\
\hline \multicolumn{4}{|c|}{ Salivary cortisol $A \cup C_{G}(\mu \mathrm{g} / \mathrm{dL} / \mathrm{min})$} \\
\hline Mean (SD) & $90.22(63.36)$ & $94.21(63.13)$ & $0.616^{* *}$ \\
\hline Median (IQR) & $76.95(82.18)$ & $82.96(67.71)$ & - \\
\hline \multicolumn{4}{|c|}{ Cortisol awakening response $(\mu \mathrm{g} / \mathrm{dL})$} \\
\hline Mean (SD) & $0.03(0.15)$ & $0.06(0.12)$ & $0.373^{* *}$ \\
\hline
\end{tabular}

SD: standard deviation; IQR: interquartile range; NA: not applicable (paired samples); NS: not significant; BMI: body mass index; $\mathrm{AUC}_{\mathrm{G}}$ : area under the curve with respect to ground (raw data). *Mann-Whitney test; **Two sample t-test. 


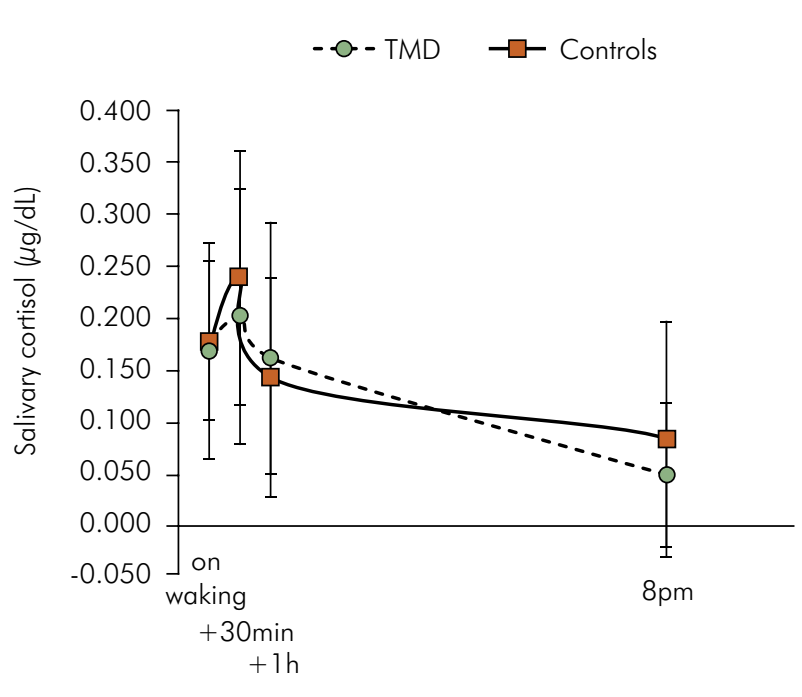

Figure 1. Salivary cortisol profiles in TMD and control groups. Graph presents means and standard deviations (raw data).

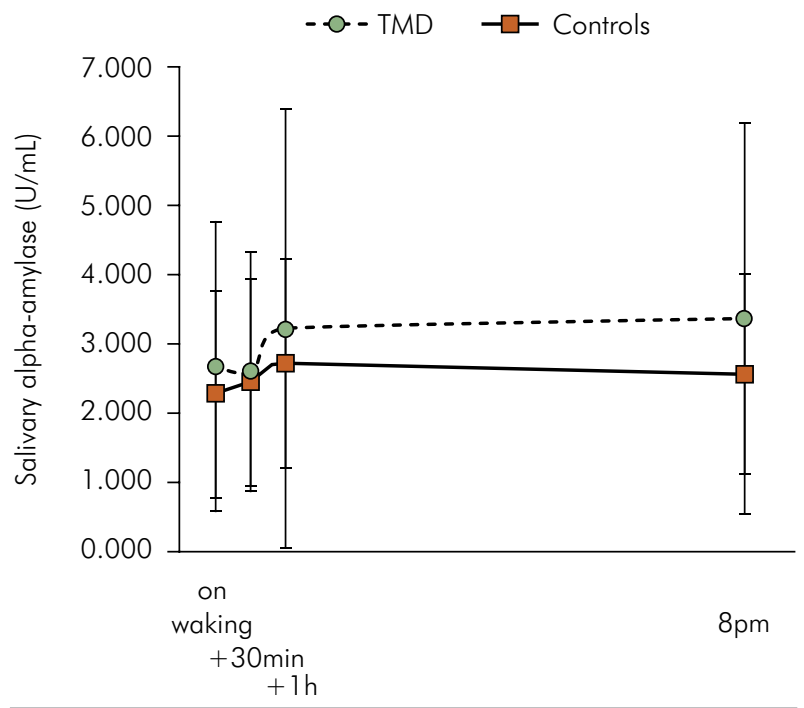

Figure 2. Salivary alpha-amylase profiles in TMD and control groups. Graph presents means and standard deviations (raw data).

Table 2. Characteristics of the sample in accordance to the evaluation of anxiety symptoms

\begin{tabular}{|c|c|c|c|c|c|c|}
\hline \multirow[t]{2}{*}{ Variable } & Social anxiety & $\begin{array}{l}\text { Separation or } \\
\text { panic }\end{array}$ & Harm avoidance & Physical symptoms & MASC total score & \multirow{2}{*}{$\begin{array}{l}\text { Range of total } \\
\text { score }\end{array}$} \\
\hline & Mean (SD) & Mean (SD) & Mean (SD) & Median (IQR) & Mean (SD) & \\
\hline TMD group & $14.7(6.3)$ & $15.1(6.0)$ & $14.0(5.4)$ & 14.0 (1 1.8) & $57.9(20.2)$ & $17-102$ \\
\hline Control group & $9.1(6.5)$ & $12.4(4.7)$ & $13.0(4.8)$ & $8.0(6.5)$ & $43.2(16.0)$ & $0-72$ \\
\hline$p$-value & $0.0003^{* *}$ & $0.0279^{* *}$ & $0.4210^{* *}$ & $0.0030^{*}$ & $0.0007^{* *}$ & - \\
\hline
\end{tabular}

SD: standard deviation; IQR: interquartile range; MASC: Multidimensional Anxiety Scale for Children. *Mann-Whitney test; ${ }^{* *}$ Two sample t-test.

\section{Discussion}

Presumably, the psychophysiological responses produced by anxiety are associated with an increased activity of the sympathetic nervous system and/or HPA axis; however, no difference was observed in the secretion profile of the salivary stress parameters between the paired study groups, whereas the TMD group scored higher in the evaluation of anxiety symptoms. The results of comparing the anxiety symptoms between children with and without TMD reported a large effect size and strong test power, which denotes the strength of these findings.

A previous study revealed more serious oral health impacts on the overall well-being and higher diurnal decline of the salivary cortisol levels in children and preadolescents with TMD when compared with the controls. ${ }^{22}$ Studies including young subjects are rare. In adults, a previous study observed that the plasma cortisol levels in 15 females with welldefined TMD were $30-50 \%$ higher than those of the controls. ${ }^{14}$ According to the authors, this increased activation of the HPA axis may be a result of the conscious perception of facial pain, which may be worse than any pain elsewhere in the body. Similarly, Da Silva et al. ${ }^{16}$ observed significant differences in the morning salivary cortisol levels between females with and without TMD, and the former also reported higher scores of depression and somatization.

No significant difference was found in the sAA levels between the clinical groups. TMD may involve dysregulation of sympathetic activity, ${ }^{19}$ as TMD patients reported lower plasma epinephrine and norepinephrine levels at baseline and in response to challenges than healthy females in a previous study, which may compromise the cardiovascular and catecholamine responses to the stressors over time. ${ }^{19}$ Alternatively, Nater et al. ${ }^{11}$ observed that chronic stress 
and stress reactivity were associated with higher sAA activity throughout the day. To the best of our knowledge, this is the first study that examined the sAA levels as a marker of sympathetic nervous system in the pediatric subjects with TMD, observing the secretion profiles in 2 days. The literature revealed that this condition may worsen over time; ${ }^{3,4}$ thus, if the signs and symptoms of TMD are associated with a dysregulation of the sympathetic activity, they need to be examined in future studies.

Both groups reported sAA secretion profiles similar to the normative data, with the lowest and highest levels found upon waking and in the late evening, respectively. We did not observe any significant correlation between the salivary cortisol and sAA levels in either group, in agreement with the previous findings in healthy adults, ${ }^{11,25}$ which reveals that this pattern does not differ in young subjects with TMD.

Corroborating the present results, Nilsson and Dahlström ${ }^{32}$ observed that, despite RDC/TMD diagnosis, female adults with TMD appeared to be more psychologically distressed than the psychometrically evaluated controls, although they did not observe any significant differences in the awakening salivary cortisol levels between groups. These authors emphasized that the fact that no difference was observed between groups in a single measurement of morning salivary cortisol level does not exclude the possibility of a dysregulation of the HPA axis in patients with TMD. Alternatively, the present study collected four samples on two alternate weekdays to examine the diurnal salivary cortisol and sAA profiles, and we found similar results in children with and without TMD.

In children aged 11-15 years who reported symptoms of TMD, Karibe et al. ${ }^{33}$ observed that the TMD group had significantly higher trait anxiety scores than the subjects in the control group, despite a weak association between the TMD symptoms and trait anxiety; however, the authors emphasized their study limitation, which used the self-report of TMD symptoms instead of a clinical examination, as did the present study.

Other studies also found a lack of concordance between the self-reported levels of anxiety and biological stress reactivity ${ }^{34}$, or a lack of relationship between cortisol and anxiety at certain time points of a stress paradigm..$^{9}$ Although these seem to be interrelated, anxiety and physiological stress are distinct constructs that may be individually activated under specific circumstances. ${ }^{9}$ In addition, TMD is a heterogeneous group of conditions including several signs and symptoms with different degrees that may or may not be reflected in the increasing biomarker stress levels. Since stress and anxiety may be essential in the onset and maintenance of the musculoskeletal disorders, ${ }^{35}$ each risk factor needs to be considered to appropriately diagnose and plan the management strategies; thus, further research is needed to clarify this possible relationship in the pediatric subjects.

Some authors have argued whether the use of swab is appropriate for examining all salivary markers; it has been reported that sAA can be measured in the saliva collected by passive drool, swabs, or microsponges without compromising the validity of the assay. ${ }^{28}$ In addition, swab is one of the few methods that offers at home sampling possibility, enabling the study of the diurnal profile of secretion. ${ }^{25,28}$ Notably, this study included only nonreferred subjects in the TMD group, which increases the external validity, and their symptoms were probably less severe than those of the referred sample.

\section{Conclusion}

Although children with TMD scored higher in the evaluation of anxiety symptoms, no difference was observed in the secretion profile of the salivary stress parameters between the paired groups of children with and without TMD.

\section{Acknowledgments}

The authors thank all the study participants. Moreover, the authors sincerely thank Mrs. Simone da Silva Costa (Clinical Analyses Laboratory of ABC Medical School, Santo André, Brazil) for her valuable contribution in the salivary analysis.

\section{Funding and support}

This study was supported by the State of São Paulo Research Foundation (FAPESP, SP, Brazil, grants n. 2010/01447-0, 2010/06016-9 and 2011/50419-2). 
Salivary stress biomarkers and anxiety symptoms in children with and without temporomandibular disorders

\section{References}

1. Barbosa TS, Miyakoda LS, Pocztaruk RL, Rocha CP, Gavião MB. Temporomandibular disorders and bruxism in childhood and adolescence: review of literature. Int J Pediatr Otorhinolaryngol. 2008;72(3):299-314. https://doi.org/10.1016/i.ijporl.2007.11.006

2. Silva CG, Pachêco-Pereira C, Porporatti AL, Savi MG, Peres MA, Flores-Mir $\mathrm{C}$ et al. Prevalence of clinical signs of intra-articular temporomandibular disorders in children and adolescents: a systematic review and meta-analysis. J Am Dent Assoc. 2016;147(1):10-8.E8. https://doi.org/10.1016/i.adaj.2015.07.017

3. Tecco S, Crincoli V, Di Bisceglie B, Saccucci M, Macrí M, Polimeni $A$ et al. Signs and symptoms of temporomandibular joint disorders in Caucasian children and adolescents. Cranio. 2011;29(1):71-9. https://doi.org/10.1179/crn.2011.010

4. Lövgren A, Häggman-Henrikson B, Visscher CM, Lobbezoo F, Marklund S, Wänman A. Temporomandibular pain and jaw dysfunction at different ages covering the lifespan: a population based study. Eur J Pain. 2016;20(4):532-40. https://doi.org/10.1002/ejp.755

5. Hongxing L, Astrøm AN, List T, Nilsson IM, Johansson A. Prevalence of temporomandibular disorder pain in Chinese adolescents compared to an age-matched Swedish population. J Oral Rehabil. 2016;43(4):241-8. https://doi.org/10.1111/joor.12366

6. Pizolato RA, Fernandes FS, Gavião MB. Speech evaluation in children with temporomandibular disorders. J Appl Oral Sci. 2011;19(5):493-9. https://doi.org/10.1590/S1678-77572011000500010

7. Kindler S, Samietz S, Houshmand M, Grabe HJ, Bernhardt $O$, Biffar $R$ et al. Depressive and anxiety symptoms as risk factors for temporomandibular joint pain: a prospective cohort study in the general population. J Pain. 2012;13(12):1188-97. https://doi.org/10.1016/i.jpain.2012.09.004

8. Kendall PC, Brady EU, Verduin TL. Cormorbidity in childhood anxiety disorders and treatment outcome. J Am Acad Child Adolesc Psychiatry 2001;40(7):787-94. https://doi.org/10.1097/00004583-200107000-00013

9. Simon DM, Corbett BA. Examining associations between anxiety and cortisol in high functioning male children with autism. J Neurodev Disord. 2013;5(1):32. https://doi.org/10.1186/1866-1955-5-32

10. Endler NS, Parker JDA. Stress and anxiety: conceptual and assessment issues. Stress Health. 1990;6(3):243-8. https://doi.org/10.1002/smi.2460060310

11. Nater UM, Rohleder N, Schlotz W, Ehlert U, Kirschbaum C. Determinants of the diurnal course of salivary alpha-amylase. Psychoneuroendocrinology 2007;32(4):392-401. https://doi.org/10.1016/i.psyneven.2007.02.007

12. Manfredini D, Landi N, Bandettini Di Poggio A, Dell'Osso L, Bosco M. A critical review on the importance of psychological factors in temporomandibular disorders. Minerva Stomatol. 2003;52(6):321-30.
13. Grøholt EK, Stigum H, Nordhagen R, Köhler L. Recurrent pain in children, socio-economic factors and accumulation in families. Eur J Epidemiol. 2003;18(10):965-75. https://doi.org/10.1023/A:1025889912964

14. Korszun A, Hinderstein B, Wong M, Peterson LJ. Comorbidity of depression with chronic facial pain and temporomandibular disorders. Oral Surg Oral Med Oral Pathol Oral Radiol Endod. 1996;82(5):496-500. https://doi.org/10.1016/S1079-2104(96)80192-2PMID:8936511

15. Clow A, Thorn L, Evans P, Hucklebridge F. The awakening cortisol response: methodological issues and significance. Stress. 2004;7(1):29-37. https://doi.org/10.1080/10253890410001667205

16. Andrade AS, Gamero GH, Pereira LJ, Zanin ICJ, Gavião MB. Salivary cortisol levels in young adults with temporomandibular disorders. Minerva Stomatol. 2008;57(3):109-16.

17. Skosnik PD, Chatterton RT, Swisher T, Park S. Modulation of attentional inhibition by norepinephrine and cortisol after psychological stress. Int J Psychophysiol. 2000;36(1):59-68. https://doi.org/10.1016/S0167-8760(99)00100-2

18. Inagaki T, Miyaoka T, Okazaki S, Yasuda H, Kawamukai T, Utani E et al. High salivary alpha-amylase levels in patients with schizophrenia: A pilot study. Prog Neuropsychopharmacol Biol Psychiatry. 2010;34(4):688-91. https://doi.org/10.1016/i.pnpbp.2010.03.024

19. Light KC, Bragdon EE, Grewen KM, Brownley KA, Girdler SS, Maixner W. Adrenergic dysregulation and pain with and without acute beta-blockade in women with fibromyalgia and temporomandibular disorder. J Pain. 2009;10(5):542-52. https://doi.org/10.1016/i.jpain.2008.12.006

20. Monaco A, Cattaneo R, Mesin L, Ciarrocchi I, Sgolastra F, Pietropaoli D. Dysregulation of the autonomous nervous system in patients with temporomandibular disorder: a pupillometric study. PLoS One. 2012;7(9):e45424. https://doi.org/10.1371/journal.pone.0045424

21. Koray M, Dülger O, Ak G, Horasanli S, Uçok A, Tanyeri H et al. The evaluation of anxiety and salivary cortisol levels in patients with oral lichen planus. Oral Dis 2003;9(6):298-301. https://doi.org/10.1034/j.1601-0825.2003.00960.x

22. Barbosa TS, Castelo PM, Leme MS, Gavião MB. Associations between oral health-related quality of life and emotional statuses in children and preadolescents. Oral Dis. 2012;18(7):639-47. https://doi.org/10.1111/j.1601-0825.2012.01914.x

23. Dworkin SF, LeResche L. Research diagnostic criteria for temporomandibular disorders: review, criteria, examinations and specifications, critique. J Craniomandib Disord 1992;6(4):301-55.

24. Strahler J, Berndt C, Kirschbaum C, Rohleder N. Aging diurnal rhythms and chronic stress: distinct alteration of diurnal rhythmicity of salivary alpha-amylase and cortisol. Biol Psychol 2010;84(2):248-56. https://doi.org/10.1016/j.biopsycho.2010.01.019 
25. Castelo PM, Barbosa TS, Pereira LJ, Fonseca FL, Gavião MB. Awakening salivary cortisol levels of children with sleep bruxism. Clin Biochem 2012;45(9):651-4. https://doi.org/10.1016/i.clinbiochem.2012.03.013

26. American Academy of Sleep Medicine - AASM. International classification of sleep disorders. 3rd ed. Darien, IL: American Academy of Sleep Medicine; 2014.

27. Araujo DS, Marquezin M, Barbosa TS, Fonseca F, Fegadolli C, Castelo PM. Assessment of quality of life, anxiety, socio-economic factors and caries experience in Brazilian children with overweight and obesity. Int J Dent Hyg. 2016 Oct 4. https://doi.org/10.1111/idh.12248

28. Granger DA, Kivlighan KT, el-Sheikh M, Gordis EB, Stroud LR. Salivary alpha-amylase in biobehavioral research: recent developments and applications. Ann N Y Acad Sci. 2007;1098(1):122-44. https://doi.org/10.1196/annals.1384.008

29. Campos MM, Kobayashi FY, Barbosa TS, Costa SS, Lucas BL, Castelo PM. Characteristics of salivary secretion in normal-weight, overweight and obese children: a preliminary study: salivary composition and excessive fat tissue. Odontology. 2014;102(2):318-24. https://doi.org/10.1007/s10266-013-0103-8

30. Gordis EB, Granger DA, Susman EJ, Trickett PK. Asymmetry between salivary cortisol and alpha-amylase reactivity to stress: relation to aggressive behavior in adolescents. Psychoneuroendocrinology. 2006;31(8):976-87. https://doi.org/10.1016/i.psyneven.2006.05.010
31. Dunst C, Hamby DW, Trivette CM. Guidelines for calculating effect sizes for practice-based research syntheses.

Centerscope. 2004;2(2):1-10.

32. Nilsson AM, Dahlström L. Perceived symptoms of psychological distress and salivary cortisol levels in young women with muscular or disk-related temporomandibular disorders. Acta Odontol Scand 2010;68(5):284-8. https://doi.org/10.3109/00016357.2010.494620

33. Karibe H, Shimazu K, Okamoto A, Kawakami T, Kato Y, Warita-Naoi S. Prevalence and association of self-reported anxiety, pain, and oral parafunctional habits with temporomandibular disorders in Japanese children and adolescents: a cross-sectional survey. BMC Oral Health. 2015;15(1):8. https://doi.org/10.1186/1472-6831-15-8

34. Hare OA, Wetherell MA, Smith MA. State anxiety and cortisol reactivity to skydiving in novice versus experienced skydivers. Physiol Behav. 2013;118:40-4. https://doi.org/10.1016/i.physbeh.2013.05.011

35. Pizolato RA, Freitas-Fernandes FS, Gavião MB. Anxiety/depression and orofacial myofacial disorders as factors associated with TMD in children. Braz Oral Res. 2013;27(2):156-62. https://doi.org/10.1590/S1806-83242013000100021 